\title{
模拟体内微环境篮选与肿瘤细胞作用的 中药活性成分
}

\author{
牟朝丽，祁小妮，张婧，张志琪* \\ 药用资源与天然药物化学教育部重点实验室; 陕西师范大学化学化工学院, 西安 710062 \\ *通讯作者, E-mail: zqzhang@ @snnu.edu.cn \\ 收稿日期: 2013-06-06; 接受日期: 2013-08-13; 网络版发表日期: 2013-11-11 \\ doi: 10.1360/032013-203
}

\begin{abstract}
摘要利用三维(3D)细胞反应器模拟体内微环境, 建立了一种与肿瘤细胞作用的活性分 子的䇻选和分析方法. 利用药物与三维细胞反应器中活肿瘤细胞和固化肿瘤细胞分别作用 后的 HPLC 生物指纹谱峰面积之间有无显著性差异, 建立了与细胞结合的活性成分的䇻选 识别模型. 已知抗肿瘤药物紫杉醇和白䓠声醇的谱峰均具有显著性差异, 而非抗肿瘤药物酮 洛芬和青霉素 $G$ 的谱峰均没有显著性差异, 证明利用该模型筤选识别与细胞结合的活性成 分是可行的. 此外, 应用该模型从中草药桃儿七提取物中䇻选出了 7 种可作用于 Lovo 细胞 的活性成分. 此研究提供了一种模拟体内微环境下与肿瘤细胞作用的活性成分的笁选和分 析方法, 在药物发现环节, 特别是中草药活性成分研究中具有潜在的应用价值.
\end{abstract}

\section{关键词}

体内微环境 $3 \mathrm{D}$ 细胞反应器 特异性结合 中草药 药物篮选

\section{1 引言}

中药是我国传统医学的有益组成部分, 是抗肿 瘤制剂的重要来源. 很多中药药物是由中药粗提物 制备而成, 然而, 中药粗提物大部分含有成百上千种 类型、结构和含量不同的成分, 其有效成分通过多成 分、多靶点、多途径发挥药效. 这给药物笁选带来了 极大的困难, 使抗肿瘤药物的篎选成为研究中药抗 肿瘤过程的关键环节. 目前, 关于荧光蛋白 ${ }^{[1]}$ 、微管 蛋白 ${ }^{[2]} 、 \mathrm{G}$-蛋白偶联受体 ${ }^{[3]}$ 、细胞膜 ${ }^{[4]}$ 、活细胞 ${ }^{[5 \sim 8]}$ 等与生物活性成分作用的文献已有报道. 值得关注 的是, 由于细胞更接近体内生理环境, 基于细胞的药 物篎选方法, 具有特异性高、可操作性强、可高通量 等优点, 已被广泛应用于药物靶点确证、吸收、分布、 代谢和毒性研究 ${ }^{[9]}$. 另外, 在药物笁选的不同阶段, 已出现了基于活细胞的微型和纳米级的分析技 术 ${ }^{[10 \sim 14]}$.
在体内环境下, 所有的细胞均处于三维(3D)空 间之中. 二维 $(2 \mathrm{D})$ 培养的细胞, 缺乏 $3 \mathrm{D}$ 细胞支架来 支持细胞生长和维持适当的组织功能, 不能模拟体 内微环境. 3D 培养的细胞, 可以重现细胞-细胞和细 胞-基质之间的相互作用, 更能真实地反映细胞生长 的微环境 ${ }^{[15]}$. 在药物功效预测方面, 一个更接近体内 生理条件的体外模型能提供更可靠的数据 ${ }^{[16]}$. 因此, 细胞在 3D 条件下的响应更能代表其在体内条件下的 响应, 基于 $3 \mathrm{D}$ 细胞的药物笁选方法将更有效.

本研究旨在利用 $3 \mathrm{D}$ 细胞反应器模拟体内微环境, 建立一种与肿瘤细胞作用的活性药物分子的篮选和 分析方法: (1) 利用本实验室自制的丝素蛋白/胶原蛋 白/纳米羟基磷灰石 $(\mathrm{SCH})$ 复合多孔支架 ${ }^{[17,18]}$, 构建 了一个 $3 \mathrm{D}$ 细胞反应器; (2) 将人结肠癌细胞系 Lovo 细胞动态接种和灌流培养于 $3 \mathrm{D}$ 细胞反应器中的 $\mathrm{SCH}$ 复合多孔支架上，并完成药物-细胞相互作用; (3) 富 
集药物细胞作用后的流出液, 用 HPLC 分析检测, 篣选与肿瘤细胞特异性结合的成分. 结果表明, 这一 方法具有高效、简便等特点, 可用于与细胞特异性结 合成分的篎选和分析, 以及中药中候选药物的篎选.

\section{2 实验部分}

\section{1 仪器与试剂}

XO-SM200 超声微波联用仪(中国南京先欧仪器 制造有限公司); Quanta200 环境扫描电子显微镜 (SEM, 日本 FEI 公司); Nikon TE2000 倒置相差显微 镜(IM, 日本 Nikon 公司); Leica TCS SP5 激光共聚焦 扫描显微镜(CLSM, 德国 Leica 公司); LC-MS 系统包 括 Agilent 1100 LC(美国 Agilent 公司)和 Bruke Q6000 MS (德国 Bruke 公司); 冷冻干燥机(德国 CHRIST 公 司); $\mathrm{CO}_{2}$ 捊育箱(德国 Heraeus 公司); SW-CJ-IF 型超净 台 (中国苏州安泰空气技术有限公司); Millicell-PCF 超纯水机(美国 Millipore 公司).

胎牛血清、DMEM、胰酶和 DMSO 购于 SigmaAldrich 公司; 戊二醛购于 Amersco 公司; 乙腈、甲醇 (HPLC 级)购于 Fisher Scientific 公司; 白称芦醇、紫 杉醇标准品购于中国药品生物制品检定所.

$\mathrm{SCH}$ 复合多孔支架由本实验室自制 ${ }^{[17,18]}$; 人结 肠癌细胞系 Lovo 细胞购自中国典藏物培养中心(武 汉); 桃儿七购于西安药材市场, 经陕西师范大学生 命科学学院任毅教授鉴定.

\section{2 药品供试液的制备}

分别将白萑芦醇、紫杉醇、酮洛芬、青霉素 $\mathrm{G}$ 用 DMSO 溶解(DMSO 最终浓度不能超过 $0.1 \%$ ), 经 $0.22 \mu \mathrm{m}$ 滤膜过滤除菌后用 PBS 稀释至 $15 \mu \mathrm{g} / \mathrm{mL}$.

将桃儿七粉碎, 过篮. 用甲醇在微波功率 $400 \mathrm{~W}$, 提取温度 $50{ }^{\circ} \mathrm{C}$ 条件下提取 2 次, 每次 $15 \mathrm{~min}$. 提取 液经旋转蒸发仪浓缩后冷冻干燥, 用 DMSO 溶解 (DMSO 最终浓度不能超过 $0.1 \%$ ), 经 $0.22 \mu \mathrm{m}$ 滤膜过 滤除菌后用 PBS 稀释至 $15 \mu \mathrm{g} / \mathrm{mL}$.

\section{3 色谱分析}

药物的 HPLC 分析在 Shimadzu-10A 液相色谱仪 (主要由 1 个 SPD-10A VP 紫外检测器, 2 个 $10 \mathrm{~A}$ VP 葲, 1 个 $7725 \mathrm{i}$ 进样阀, 1 个 HT-220A 柱温箱以及 N2000 工作站组成)上进行. 色谱分离在 Agilent
SB-C $\mathrm{C}_{18}$ 色谱柱上完成, 检测条件如下: 柱温 $25{ }^{\circ} \mathrm{C}$, 流动相流速 $1.0 \mathrm{~mL} / \mathrm{min}$, 进样体积 $20 \mu \mathrm{L}$. 对于紫杉 醇分析, 流动相为乙腈(A)-水(B) (60:40), 检测波长 为 $227 \mathrm{~nm}$; 对于白藜芦醇分析, 流动相为甲醇 (A) $-0.5 \%$ 甲酸水溶液(B) (45:55), 检测波长为 $303 \mathrm{~nm}$; 对于酮洛芬分析, 流动相为乙腈 $(\mathrm{A})-0.02 \mathrm{~mol} / \mathrm{L} \mathrm{pH}$ $3.5 \mathrm{KH}_{2} \mathrm{PO}_{4}$ 水溶液(B) $(60: 40)$, 检测波长为 $254 \mathrm{~nm}$; 对于青霉素 $\mathrm{G}$ 分析, 流动相为 $(\mathrm{A})-0.02 \mathrm{~mol} / \mathrm{L} \mathrm{pH} 2.5$ $\mathrm{KH}_{2} \mathrm{PO}_{4}$ 水溶液 (B) (10:90), 检测波长为 $220 \mathrm{~nm}$; 对 于桃儿七分析, 流动相为乙腈(A)- $0.04 \%$ 甲酸水溶液 (B), 洗脱梯度为 0 20 min 90\% 77\% B、20 40 min $68 \% \mathrm{~B} 、 40 \sim 65 \min 59 \% \mathrm{~B}$, 检测波长为 $290 \mathrm{~nm}$.

采用 Ultimate 3000 HPLC 耦合 maXisTM UHRTOF 质谱仪(含 ESI 源) 对桃儿七提取液中的成分进行 鉴定. 质谱分析条件为: 采用高纯氦气( $99.999 \%)$ 为 碰撞气, 高纯氮气( $99.999 \%)$ 为雾化气. 分别采用正 离子模式和负离子模式分析. 质谱参数为: 扫描范围 $m / z=120 \sim 1000$, 雾化气压力为 $0.8 \mathrm{bar}$, 干燥气流速 为 $8.0 \mathrm{~L} / \mathrm{min}$, 干燥气温度为 $180{ }^{\circ} \mathrm{C}$, 毛细管电压为 $4000 \mathrm{~V}$.

\section{4 细胞悬液的制备}

将 Lovo 细胞置于 $25 \mathrm{~cm}^{2}$ 的细胞培养瓶中, 用含 $10 \%(v: v)$ 胎牛血清的 DMEM 细胞培养液在 $37{ }^{\circ} \mathrm{C}$ 、 $5 \%$ 二氧化碳、 $95 \%$ 相对湿度的二氧化碳培养箱中培 养, 每 2 天换液一次. 待细胞长至 $80 \%$ 汇合度时, 使 用胰蛋白酶消化液(含 $0.25 \%$ 胰酶和 $0.04 \%$ EDTA) 消 化细胞, $1200 \mathrm{r} / \mathrm{min}$ 离心 $3 \mathrm{~min}$, 弃上层清液, 加入适 量培养液稀释细胞, 得 $1 \times 10^{6}$ 个细胞 $/ \mathrm{mL}$ 的细胞 悬液.

\section{$2.53 \mathrm{D}$ 细胞反应器的构建}

$3 \mathrm{D}$ 细胞反应器(图 1)主要由具有 $30^{\circ}$ 斜坡的 $\mathrm{SCH}$ 复合多孔支架、反应器外壳体、反应器内壳体、密封 上盖、支撑板、进气管、出气单向阀门、进液管、防 水透气膜、出液管、蠕动原、细胞悬液瓶、缓冲液瓶、 细胞培养液瓶、废液瓶及阀门组成, 通过 $\mathrm{Co}^{60}$ 辐照 灭菌.

在反应器外壳体内安装有反应器内壳体, 反应 器外壳体和反应器内壳体采用有机玻璃制成, 形状 为圆柱形。二者之间保持有间隙, 形成水流通道, $37{ }^{\circ} \mathrm{C}$ 的循环水从反应器外壳体与反应器内壳体之间 


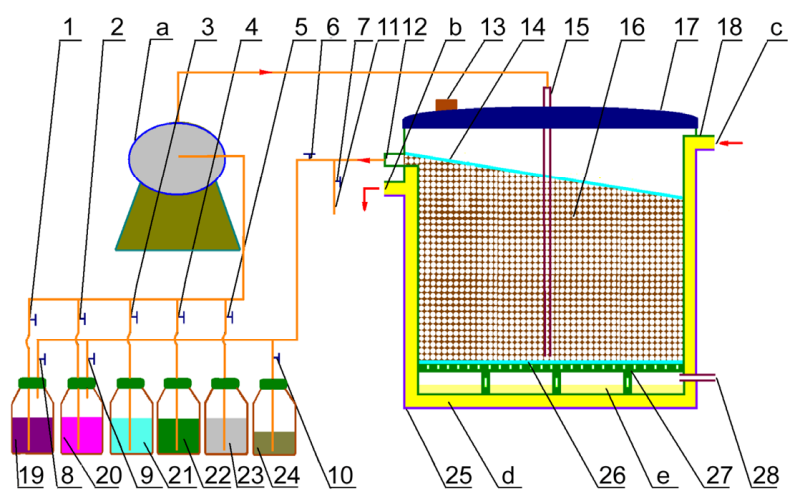

图 $13 \mathrm{D}$ 细胞反应器. (a) 蠕动原; (b, c) $37{ }^{\circ} \mathrm{C}$ 循环水出口和 进口; (d) $37{ }^{\circ} \mathrm{C}$ 循环水; (e) 无菌水; (1 10) 阀; (11) 药物溶 液出口; (12) 液体出口; $(13)$ 放气单向阀; $(14,26)$ 防水透气 膜; (15) 进液管; (16) SCH 支架; (17) 密封上盖; (18) 内壳体; （19）细胞悬液；(20) DMEM; (21) 细胞固定液(70\%乙醇); (22) 药物溶液; (23) PBS; (24) 废液; (25) 外壳体; (27) 支撑 板; (28) 进气管

的进水口进入, 流经水流通道, 从出水口流出. 反应 器外壳体的左侧下部安装有进气管, 进气管插入到 反应器外壳体内, 进气管的内端伸入到反应器内壳 体内的下部.

在反应器内壳体的上端通过螺纹连接安装有密 封上盖, 其上通过螺纹连接安装出气单向阀门. 反应 器内壳体的右侧有出液管, 出液管的外端部为加工 有孔的封闭管, 出液管与反应器内壳体相连通. 反应 器内壳体内的下部安装有支撑板, 支撑板上加工有 通气孔, 支撑板与反应器内壳体内底部之间保持一 定的距离, 形成气室. 气室与进气管相连通, 气室内 底部装有用于提供湿度的无菌水, 无菌的 $5 \%$ 二氧化 碳/ $95 \%$ 空气的混合气可进入到气室内, 从密封上盖 上的出气单向阀门流出. 支撑板上放置一张防水透 气膜, 用于通气和防止水流下. 反应器内壳体防水透 气膜上部装有 $\mathrm{SCH}$ 支架, 支架的上表面也放置一张 用于保湿通气的防水透气膜. 进液管的下端插入到 反应器内壳体内穿过 $\mathrm{SCH}$ 支架至下防水透气膜的上 表面, 进液管通过导管与蠕动百的出口相连通, 蠕动 洜的进口通过安装在导管上的阀门与细胞悬液瓶、细 胞培养液瓶、PBS 瓶、细胞固定液瓶、药物溶液瓶等 相连通, 可将它们注入到 $\mathrm{SCH}$ 支架上并循环流动. 废液通过安装在导管上的废液阀门与出液管相连通, 废弃溶液经安装在导管上的废液阀门流入到废液 瓶内.

\section{6 药物-细胞相互作用}

药物-细胞相互作用在图 1 所示的 3D 细胞反应器 中进行, 具体步骤如下: (1) 动态接种. 打开阀 $1 、 6$ 和 8 , 将细胞悬液以 $3 \mathrm{~mL} / \mathrm{min}$ 的流速循环泵入支架, 该过程持续 $20 \mathrm{~min}$ 以完成细胞接种, 动态接种完成 后, 静置 $3 \mathrm{~h}$, 使细胞在支架上黏附; (2) 淋洗. 打开 阀 2、6 和 10 , 将 DMEM 以 $1.5 \mathrm{~mL} / \mathrm{min}$ 的流速洜入, 以淋洗掉在支架上未黏附的细胞和黏附不牢固的细 胞, 这一过程持续到细胞反应器流出液中无细胞流 出为止; (3) 动态培养. 打开阀 2、6 和 9, 将 DMEM 以 $1 \mathrm{~mL} / \mathrm{min}$ 的流速泵入支架中, 此过程保持 $24 \mathrm{~h}$, 以 完成细胞的动态培养; (4) 药物-细胞相互作用. 打开 阀 6、8 和 9 , 将药物溶液以 $1 \mathrm{~mL} / \mathrm{min}$ 的流速泵入支 架中, 此过程保持 $3 \mathrm{~min}$, 以确保药物和细胞完全接 触, 静置一段时间, 完成药物和细胞的相互作用; (5) 洗脱. 打开阀 7、10 和 11 , 将 PBS 以 $3 \mathrm{~mL} / \mathrm{min}$ 的流 速洜入支架中, 将没有和细胞结合的药物洗脱出来. 将洗脱下来的药物富集到富集柱 $\left(\mathrm{C}_{18}\right.$ Sep-Pak 柱)上, 再用色谱流动相将富集柱中富集的药物冲洗下来, 用 HPLC 进行分析检测. 将药物分别与活肿瘤细胞和 固化细胞作用, 在与固化细胞作用之前, 需完成细胞 固定化过程, 打开阀 3、6 和 10, 将细胞固定液 $(70 \%$ 乙醇)百入淹没并浸泡支架 $30 \mathrm{~min}$.

\section{7 不同培养方式对细胞形貌的影响}

分别利用 IM 和 E-SEM 观察 2D 和 3D 培养的 Lovo 细胞形貌. 对于 E-SEM 分析, 分别于细胞接种 后 $1 、 3$ 和 $7 \mathrm{~d}$ 取出细胞/支架复合物, 用 PBS 清洗支 架 3 次, 用 $2.5 \%$ 戍二醛于 $4{ }^{\circ} \mathrm{C}$ 固定 $30 \mathrm{~min}$ 后, 再用 PBS 清洗, 用外科手术刀片切片, 用体积分数为 $50 \% 、 60 \% 、 70 \% 、 80 \% 、 90 \% 、 95 \% 、 100 \%$ 的乙醇 梯度脱水(每个梯度脱水 $30 \mathrm{~min})$, 喷金后用 E-SEM 观察.

\section{3 结果与讨论}

\section{1 细胞在 SCH 支架上的形态}

Lovo 细胞在 SCH 支架上的形态如图 2 所示. 由 图 2(a)可以看出, 2D 培养的 Lovo 细胞呈平面伸展状 态. 图 2(b d) 表明, 3D 培养的 Lovo 细胞呈圆形、簇 状均匀地分布在 $\mathrm{SCH}$ 支架上, 并按支架的物理形状 


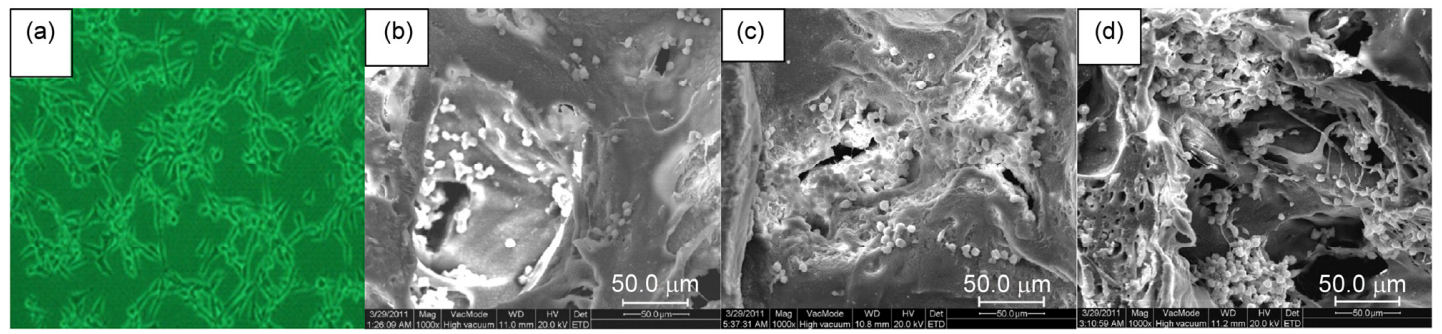

图 2 不同培养方式下 Lovo 细胞形貌图. (a) 2D 培养的 Lovo 细胞的 IM 图 ( $\times 40$ 倍); (b d) 3D 培养 1、3 和 $7 \mathrm{~d}$ 时 Lovo 细胞 的 SEM 图

排列; 支架上细胞密度随着培养时间的延长而明显 增大, 细胞表面的伪足和微线毛均表明细胞生长状 态良好 ${ }^{[19]}$. 由于 $2 \mathrm{D}$ 培养时细胞平铺在培养瓶或培养 板上, 没有传质的限制; 而 3D 培养时, 细胞培养基 质对细胞营养物质的限制和细胞的异质性使 3D 培养 具有传质局限性, 不仅会得到增殖细胞和非增殖细 胞, 而且含有坏死细胞, 这与实体瘤的多重表型相 似 ${ }^{[20]}$. 因此, 3D 培养更能模拟体内微环境.

\section{2 可行性分析}

根据药物-细胞的相互作用, 可以将药物分为两 类 ${ }^{[21]}$ : 与细胞结合的药物和与细胞不结合的药物. 其中, 与细胞不结合的药物可以被收集到富集柱上, 这些药物被认为没有抗癌作用; 相应地, 它们的生物 指纹谱峰面积在与细胞结合前后几乎没有变化. 与 细胞结合的药物则不能被收集到富集柱上, 它们的 生物指纹谱峰面积会减小. 药物与细胞的结合分为 特异性结合和非特异性结合. 由于细胞膜可以非特 异性地结合一些药物, 细胞培养支架也可能吸附一 些药物, 而这些非特异性的结合和吸附均会引起假 阳性的结果. 因此, 将药物与固化肿瘤细胞作用作为 对照, 可以排除假阳性结果. 特异性结合的成分可以 通过比较药物与活肿瘤细胞和固化肿瘤细胞作用后 的生物指纹谱峰面积而识别. 这些特异性结合的药 物的结合度可按下式计算:

$$
\text { 结合度 }=\left(A_{\mathrm{f}}-A_{\mathrm{l}}\right) / A_{\mathrm{f}} \times 100 \%
$$

式中, $A_{1}$ 和 $A_{\mathrm{f}}$ 分别为药物与活肿瘤细胞和固化肿瘤细 胞作用后的生物指纹谱峰面积.

紫杉醇和白苝芦醇是抗肿瘤药物, 具有抗人结 肠癌的效果 ${ }^{[22,23]}$, 选择为与 Lovo 细胞作用的抗肿瘤 模型药物; 酮洛芬和青霉素 $\mathrm{G}$ 是已知的非抗肿瘤药

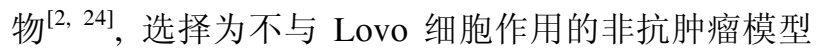

药物.

紫杉醇和白溗芦醇与活肿瘤细胞作用后的生物 指纹谱峰面积和与固化肿瘤细胞作用后的谱峰面积 之间有显著性差异 $(P<0.05)$, 根据式(1)计算, 紫杉 醇和白藜芦醇与 Lovo 细胞的结合度分别为 $64.8 \% \pm$ $6.3 \%$ 和 $81.7 \% \pm 7.8 \%$. 而酮洛芬和青霰素 $\mathrm{G}$ 与活肿 瘤细胞作用后的谱峰面积和与固化肿瘤细胞作用后 的谱峰面积之间没有显著性差异 $(P>0.05)$.

模型抗肿瘤药物和模型非抗肿瘤药物在与肿瘤 细胞作用上的显著不同, 证明该方法可用于篮选与 细胞特异性结合的生物活性成分.

\section{3 桃儿七活性成分的篮选与分析}

将反应时间设定为 $45 \mathrm{~min}$, 桃儿七提取物浓度 设定为 $15 \mu \mathrm{g} / \mathrm{mL}$, 研究桃儿七提取物与活肿瘤细胞 和固化肿瘤细胞作用后的生物指纹谱峰面积, 结果 如图 3 所示. 试验重复进行 6 次, 结果表示为平均值 \pm RSD\%.

通过比较正离子模式和负离子模式的质谱数据 以及参考文献资料 ${ }^{[25,26]}$, 可以初步确定桃儿七中的 10 种组分 (表 1).

由于檞皮素-3-O- $\beta$-D-葡萄糖苷，山奈酚-3-O- $\beta$-D葡萄糖苷和山荷叶素 3 种组分分别与活肿瘤细胞和

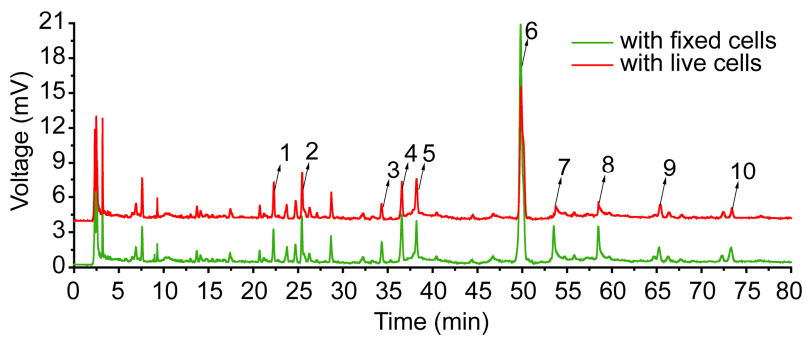

图 $315 \mu \mathrm{g} / \mathrm{mL}$ 桃儿七提取物与活肿瘤细胞和固化肿瘤细 胞作用 $45 \mathrm{~min}$ 后的生物指纹图谱 
表 1 桃儿七提取物的成分鉴定

\begin{tabular}{|c|c|c|c|}
\hline \multirow{2}{*}{ 编号 } & \multirow{2}{*}{ 成分鉴定 } & \multicolumn{2}{|c|}{ 准分子离子峰 } \\
\hline & & 负离子模式 & 正离子模式 \\
\hline 1 & 槲皮素-3-O- $\beta$-D-葡萄糖苷 & $465.138[\mathrm{M}+\mathrm{H}]^{+}$ & $463.0733[\mathrm{M}-\mathrm{H}]^{-}$ \\
\hline 2 & 山奈酚-3-O- $\beta$-D-葡萄糖苷 & $449.1099[\mathrm{M}+\mathrm{H}]^{+}$ & $447.0780[\mathrm{M}-\mathrm{H}]^{-}$ \\
\hline 3 & L-鬼臼毒素-4-O- $\beta$-D-葡萄糖苷 & $596.1704\left[\mathrm{M}+\mathrm{NH}_{4}+\mathrm{H}\right]^{+}$ & $621.1575[\mathrm{M}+\mathrm{HCOOH}-\mathrm{H}]^{-}$ \\
\hline 4 & 槲皮素 & $303.0529[\mathrm{M}+\mathrm{H}]^{+}$ & $301.0231[\mathrm{M}-\mathrm{H}]^{-}$ \\
\hline 5 & 二十六烷酸 & $396.8049[\mathrm{M}+\mathrm{H}]^{+}$ & \\
\hline 6 & 山奈酚 & $287.0583[\mathrm{M}+\mathrm{H}]^{+}$ & $285.0261[\mathrm{M}-\mathrm{H}]^{-}$ \\
\hline 7 & 鬼臼毒素 & $415.1427[\mathrm{M}+\mathrm{H}]^{+}$ & $413.1059[\mathrm{M}-\mathrm{H}]^{-}$ \\
\hline 8 & 鬼自毒酮或异鬼白苦酮 & $413.1268[\mathrm{M}+\mathrm{H}]^{+}$ & \\
\hline 9 & 山荷叶素 & $385.1248[\mathrm{M}+\mathrm{H}]^{+}$ & $383.0960[\mathrm{M}-\mathrm{H}]^{-}$ \\
\hline 10 & 去氧鬼臼毒素或 4'-去甲鬼臼毒酮 & $399.1484[\mathrm{M}+\mathrm{H}]^{+}$ & \\
\hline
\end{tabular}

固化肿瘤细胞作用后的生物指纹谱峰面积之间没有 显著性差异 $(P>0.05)$, 因此, 可以认为它们与 Lovo 细胞没有特异性结合. 而 L-鬼臼毒素-4-O- $\beta$-D-葡萄 糖苷、葪皮素、二十六烷酸、山奈酚、鬼臼毒素、鬼 臼毒酮或异鬼臼苦酮和去氧鬼臼毒素或 $4^{\prime}$-去甲鬼臼 毒酮 7 种组分分别与活肿瘤细胞和固化肿瘤细胞作 用后的生物指纹谱峰面积之间具有显著性差异 $(P<$ 0.05), 说明这 7 种组分可以与 Lovo 细胞特异性结合, 根据式(1)计算出它们的结合度分别为 $32.6 \% \pm 3.1 \%$ 、 $29.3 \% \pm 3.0 \% 、 15.2 \% \pm 1.7 \% 、 57.1 \% \pm 4.8 \% 、 68.9 \%$ $\pm 9.2 \% 、 63.6 \% \pm 8.7 \%$ 和 $46.3 \% \pm 4.8 \%$.

\section{4 结论}

本文为从天然产物和植物药物中笁选抗癌候选 药物提供了一个新的思路, 建立了一种在模拟体内 微环境下篮选和分析与肿瘤细胞特异性作用的活性 成分的新方法, 采用已知抗肿瘤药物和非抗肿瘤药 物验证了方法的可行性. 利用该方法从中草药桃儿 七提取物中笁选出了 7 种能与 Lovo 细胞特异性结合 的活性成分, 这一亲和篮选方法还可用于从多组分 复杂体系中笁选和分析与其他贴壁细胞特异性结合 的活性成分.

致谢本工作得到国家自然科学基金 $(20875060,21275098)$ 和教育部高等学校博士点学科专项科研基金 (20110202110005)资助, 特此一并致谢.

\section{参考文献}

1 Wolff M, Wiedenmann J, Nienhaus GU, Valler M, Heilker R. Novel fluorescent proteins for high-content screening. Drug Discov Today, 2006, 11: 1054-1060

2 Sun C, Fu J, He S, Pan Y. An integrative approach for the isolation, screening and analysis of antitumor agents by liquid chromatography combined with mass spectrometry. Anal Chim Acta, 2009, 655: 86-91

3 Conigrave AD, Hampson DR. Broad-spectum amino acid-sensing class C G-protein coupled receptors: Molecular mechanisms, physiological significance and options for drug development. Pharmacol Therapeut, 2010, 127: 252-260

4 Wang S, Sun M, Zhang YM, Du H, He LC. A new A431/cell membrane chromatography and online high performance liquid chromatography/mass spectrometry method for screening epidermal growth factor receptor antagonists from radix sophorae flavescentis. $J$ Chromatogr A, 2010, 1217: 5246-5252

5 Lei X, Kong L, Zou H, Ma H, Yang L. Evaluation of the interaction of bioactive compounds in cortex pseudolarix and radix stephaniae by the microdialysis probe coupled with high performance liquid chromatography-mass spectrometry. J Chromatogr A, 2009, 1216: 2179-2184

6 Zhang S, Tong W, Zheng B, Susanto TAK, Xia L, Zhang C, Ananthanarayanan A, Tuo X, Sakban RB, Jia R, Iliescu C, Chai KH, McMillian M, Shen S, Leo H, Yu H. A robust high-throughput sandwich cell-based drug screening platform. Biomaterials, 2011, 32: 1229-1241

7 Khoshmanesh K, Akagi J, Nahavandi S, Kourosh KZ, Baratchi S, Williams DE, Cooper JM, Wlodkowic D. Interfacing cell-based assays in environmental scanning electron microscopy using dielectrophoresis. Anal Chem, 2011, 83: 3217-3221

8 Kwon CH, Wheeldon I, Kachouie NN, Lee SH, Bae H, Sant S, Fukuda J, Kang JW, Khademhosseini A. Drug-eluting microarrays for 
cell-based screening of chemical-induced apoptosis. Anal Chem, 2011, 83: 4118-4125

9 El-Ali J, Sorger PK, Jensen KF. Cell on chips. Nature, 2006, 442: 403-411

10 Ostrovidov S, Annabi N, Seidi A, Ramalingam M, Dehghani F, Kaji H, Khademhosseini A. Controlled release of drugs from gradient hydrogels for high-throughput analysis of cell-drug interaction. Anal Chem, 2012, 84: 1302-1309

11 Whitesides GM. The origins and the futureof microfluidics. Nature, 2006, 442: 368-373

12 Yager P, Edwards T, Fu E, Helton K, Nelson K, Tam MR, Weigl BH. Microfluidic diagnostic technologies for global public health. Nature, 2006, 442: 412-418

13 Smith C. Tools for drug discovery: Tools of the trade. Nature, 2007, 446: 219-222

14 Dittrich PS, Manz A. Lab-on-a-chip: Microfluids in drug discovery. Nat Rev Drug Discov, 2006, 5: 210-218

15 Griffith LG, Swartz MA. Capturing complex 3D tissue physiology in vitro. Nat Rev Mol Cell Bio, 2006, 7: 211-224

16 Breslin S, O’Driscoll L. Three-dimensional cell culture: The missing link in drug discovery. Drug Discov Today, 2013, 18: 240-249

17 Mou ZL, Duan LM, Qi XN, Zhang ZQ. Preparation of silk fibroin/collagen/hydroxyapatite composite scaffold by particulate leaching method. Mater Lett, 2013, 105: 189-191

18 张志琪, 牟朝丽, 祁小妮, 段俐敏. 丝素蛋白/羟基磷灰石/胶原蛋白复合多孔支架的制备方法. 中国专利, ZL2011102418051, 2012-01-18

19 Qi XN, Mou ZL, Zhang J, Zhang ZQ. Preparation of chitosan/silk fibroin/hydroxyapatite porous scaffold and its characteristics in comparis on to bi-component scaffolds. J Biomed Mater Res A, 2014, 102: 366-372

20 Jeong SH, Lee DW, Kim S, Kim J, Ku B. A study of electrochemical biosensor for analysis of three-dimensional (3D) cell culture. Biosens Bioelectron, 2012, 35: 128-133

21 Mou ZL, Qi XN, Liu RL, Zhang J, Zhang ZQ. Three-dimensional cell bioreactor coupled with high performance liquid chromatographymass spectrometry for the affinity screening of bioactive components from herb medicine. J Chromatogr A, 2012, 1243: 33-38

22 Athar M, Back JH, Tang X, Kim KH, Kopelovich L, Bickers DR, Kim AL. Reseveratrol: A review of preclinical studies for human cancer prevent. Toxicol Appl Pharm, 2007, 224: 274-283

23 Xi G, Hu X, Wu B, Jiang H, Young CYF, Pang Y, Yuan H. Autophagy inhibition promotes paclitaxel-induced appotosis in cancer cells. Cancer Lett, 2011, 307: 141-148

24 Chan A, Tan SH, Wong CM, Yap KY, Ko Y. Supportive care matters. Clin Ther, 2009, 31: 2379-2386

25 Gurski LA, Jha AK, Zhang C, Jia X, Farach-Carson MC. Hyaluronic acid-based hydrogels as 3D matrices for in vitro evaluation of chemotherapeutic drugs using poorly adherent prostate cancer cells. Biomaterials, 2009, 30: 6076-6085

26 Feder-Mengus C, Ghosh S, Reschner A, Martin I, Spagnoli GC. New dimensions in tumor immunology: What does 3D culture reveal? Trends Mol Med, 2008, 14: 333-340 


\title{
Affinity screening and analysis of active components interacted with cancer cells at simulating the in vivo micro-environment from traditional Chinese medicines
}

\author{
MOU ZhaoLi, QI XiaoNi, ZHANG Jing, ZHANG ZhiQi* \\ Key Laboratory of Medicinal Resources and Natural Pharmaceutical Chemistry, Ministry of Education; School of Chemistry and \\ Chemical Engineering, Shaanxi Normal University, Xi'an 710062, China \\ *Corresponding author (email: zqzhang@ snnu.edu.cn)
}

\begin{abstract}
An affinity screening and analysis method was established by combining three-dimensional cell bioreactor with HPLC/MS for the active components interacted with cancer cells at simulating the in vivo micro-environment. The differences between the biological fingerprinting chromatograms of HPLC after interacting with live and fixed cells were used to establish a screening recognition model to distinguish bioactive components interacted specifically. Having significant difference $(P<0.05)$ for model anticancer drugs (paclitaxel and resveratrol) and having no significant difference $(P<0.05)$ for model non-anticancer drugs (ketoprofen and penicillin $\mathrm{G})$ with model cancer cells demonstrated the feasibility of this recognition model. The model was used to screen bioactive components from Sinopodophyllum hexandrum (Royle) Ying extract. Seven components interacted with Lovo cells were screened. This paper provides a screening and analysis method, mimicking in vivo micro-environment, for bioactive components interacted with cancer cells. This method has the potential to be used in drug discovery programs especially in bioactive components research from traditional Chinese medicines.
\end{abstract}

Keywords: in vivo micro-environment, three-dimensional cell bioreactor, specific binding, traditional Chinese medicines, drug screening 